\title{
Pressure Ulcer Risk Assessment by Monitoring Interface Pressure and Temperature
}

\author{
Farve Daneshvar Fard, Sahar Moghimi and Reza Lotfi \\ Department of Electrical Engineering \\ Biomedical Engineering Research Center, Ferdowsi University of Mashhad \\ Mashhad, Iran \\ f.daneshvarfard@yahoo.com, s.moghimi@ferdowsi.um.ac.ir, rlotfi@ieee.org
}

\begin{abstract}
Pressure ulcers are tissue damages resulting from blood flow restriction, which occur when a subject's tissue is exposed to high pressure for a lengthy period of time. These painful sores are common in bedridden and wheelchair-bound patients and the elderly. In this work, a continuous pressure and temperature monitoring system is developed for pressure ulcer prevention. The system consists of 64 pressure sensors and 64 temperature sensors on a $40 \times 50 \mathrm{~cm}^{2}$ sheet. Real time pressure and temperature data and corresponding maps are displayed on a computer simultaneously. Risk assessment can be performed by monitoring and recording pressure and temperature absolute values as well as deviations through time.
\end{abstract}

Keywords-Interface pressure and temperature monitoring; pressure ulcer; risk assessment

\section{INTRODUCTION}

Pressure ulcers are tissue damages of particular areas of body resulted from prolonged exposure to high pressure. In long term, the applied pressure restricts blood flow which leads to tissue breakdown. Pressure ulcers can occur in all situations where subjects are in a lengthy pressure-bearing position, but they are more probable in bedridden patients or those who are wheelchair-bound.

Process of maintenance and treatment of the people who developed pressure ulcer is a burden for the community in terms of economy and healthcare. Pressure ulcers multiply the expenditure by increasing the length of being hospitalized up to several times. Estimated price of managing a fullthickness pressure ulcer equals $\$ 70000$ and in the U.S., there is an annual cost of around $\$ 11$ billion for treating pressure ulcers [1].

Today, inspite of so many attempts for preventing pressure ulcers, there is not a clear and significant method for solving the issue. In 1950, the simplest method of preventing pressure ulcers was presented by a British nurse, which was repositioning of immobile patients every two hours [2]. Today, there are different types of pressure-reducing devices for unmoved patients. Static systems reduce interface pressure by distributing it over a larger area and dynamic support surfaces dispense alternating currents of air to different points of the contact surface. However, all of these methods are not necessarily satisfactory in practice. There are not enough guarantees that pressure will be relieved from the risk area by the repositioning method. Using static devices does not work alone and patients can still develop pressure sores if there is not enough care. Furthermore, dynamic surfaces are useless on a large scale due to their excess cost, hence extended studies, further attempts and newer methods are required.

Recent approaches for preventing pressure ulcers are based on measuring interface pressure. That is the only way we can estimate the pressure applied on each part of the body tissue and so prevent increasing pressure and developing sores.

Reswick and Roger [3] investigated the relationship between pressure and time. High pressures are tolerable for short times only and, if unrelieved, will lead to tissue necrosis [4]. Low pressures, however, are damaging if left unrelieved for a long period of time [5]. Sakai, et al. [6] performed continuous monitoring of interface pressure distribution in intensive care patients which gives good information about the relationship between intensity and duration of the applied pressure for pressure ulcer prevention.

Using electro-pneumatic sensors for measuring interface pressure, Meffre, et al. [7] designed a particular type of seat for wheelchair-bound patients. These kinds of pressure sensors are more expensive than capacitive and resistive sensors and slower in data acquisition. Yip, et al. [8] presented a flexible pressure monitoring system. The prototype consists of capacitive pressure sensors on a $17 \times 22$ $\mathrm{cm}^{2}$ sheet.

Yang, et al. [9] designed and evaluated an air-alternating wheelchair seat. They used resistive pressure sensors for measuring interface pressure. Drennan and Southard [10] presented a system consisting of pressure sensitive pads 
which produces alarms if the pressure intensity is more than the threshold adjusted by the user.

In this paper, we present a system for continuous monitoring of interface pressure and temperature. In comparison with previous works, one part of the novelty is about including temperature sensors and measuring pressure and temperature of different points simultaneously. According to National Pressure Ulcer Advisory Panel's definition, temperature is identified as an indicator of pressure ulcer development and it can help us in recognizing risk situations. Both increased and decreased skin temperature can be used for indicating pressure ulcer development, however, a tissue breakdown may occur even if there is no temperature changes [11].

The remainder of this paper is structured as follows. Section II describes the pressure and temperature sensing approaches. System design, including sensors array setup as well as, circuit and software design, is explained in section III. In section IV we present the experimental results. Conclusions are given in section $\mathrm{V}$.

\section{STRATEGY AND APPROACH}

\section{A. Pressure Sensing}

In this work pressure sensing is carried out by Interlink Electronics force sensing resistors (FSR-part no. 400), which exhibit a decrease in resistance with an increase in the force applied to the active surface. By measuring the resistance, the applied force can be extracted and therefore the corresponding pressure value can be easily calculated. The force vs. resistance characteristic of the FSR, available in documents, provides an overview of FSR typical response behavior. To have a more precise measurement of force, we calibrated each sensor we used by applying specific values of force and measuring the corresponding values of resistance. The resistance vs. force characteristic of a sample sensor resulted from calibration is given in Fig. 1. The other sensors behave almost the same.

\section{B. Temperature Sensing}

The Dallas Semiconductor DS18B20 digital thermometer is used for sensing temperature. The DS18B20 communicates over a 1-wire bus that by definition requires only one data line (and ground) for communication with a central microprocessor.

\section{SYSTEM DESIGN}

\section{A. Sensors Array Setup}

To sense pressure and temperature over a large area, an array of sensors is required. We used an array of $8 \times 16$ sensors to cover an area of $30 \times 40 \mathrm{~cm}^{2}$. Each row of the array consists of 8 pressure sensors and 8 temperature sensors as illustrated in Fig. 2. Therefore the setup includes 64 pressure sensors and 64 temperature sensors; all fixed on a Plexiglas sheet of size $40 \times 50 \mathrm{~cm}^{2}$. A PCB of the same size was designed for wiring the sensors and is fixed under the Plexiglas sheet.

\section{B. Circuit Design}

The sensors array setup, covered with a thin mattress, is placed under the patient. Measuring the resistances of FSR sensors is carried out by an Atmel ATMEGA16 microcontroller analog-to-digital converter. The same microcontroller controls multiplexers to select one resistive sensor at any time according to a particular sequence. The entire array of the resistive sensors is scanned every $320 \mathrm{~ms}$ with a sampling rate of $3 \mathrm{~Hz}$.

A simplified schematic of resistive sensors array and the related electronic circuitry is shown in Fig. 3. Current source, designed by LM324 operational amplifier and 2N3906 transistor, sources a current of $100 \mu \mathrm{A}$ to the selected resistor and the ADC measures the corresponding value of voltage proportional to the resistance value. Each sensor is placed in a serial connection with a diode to prevent current flows into other sensors and as a result creating undesirable routs. Reading digitized temperature is performed by another Atmel ATMEGA16 microcontroller. Moreover, this microcontroller controls multiplexer to select each row of temperature sensors according to an appropriate order. A simplified schematic of temperature sensors and the electronics is shown in Fig. 4. The designed PCB, illustrated in Fig. 5, interfaces the electronics and the sensors sheet.

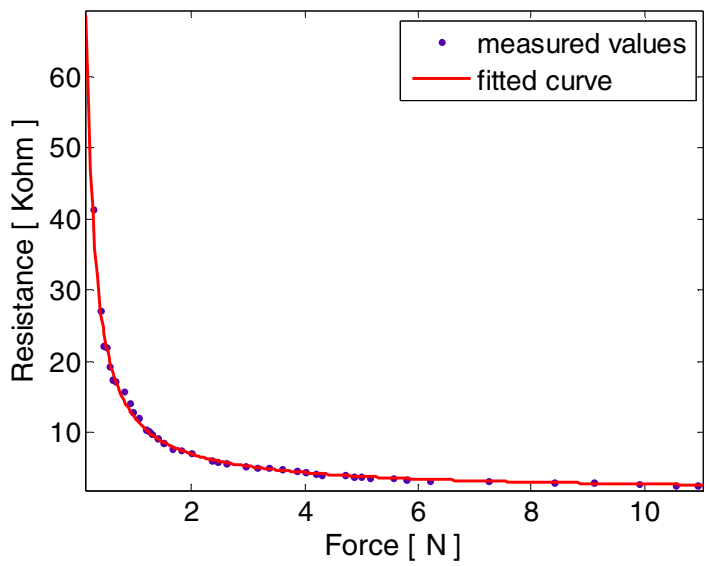

Figure 1: Resistance vs. force characteristic of a sample FSR. Dots: measured values, solid line: fitted curve.

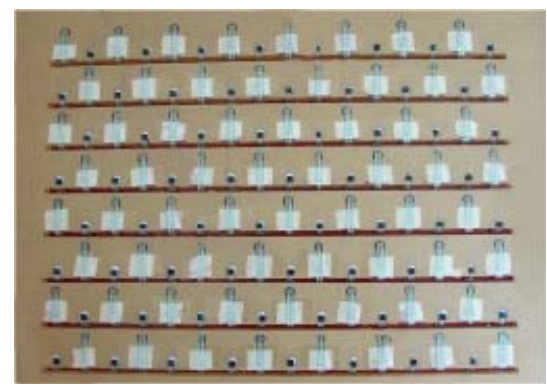

Figure 2: Pressure and temperature sensors array. 


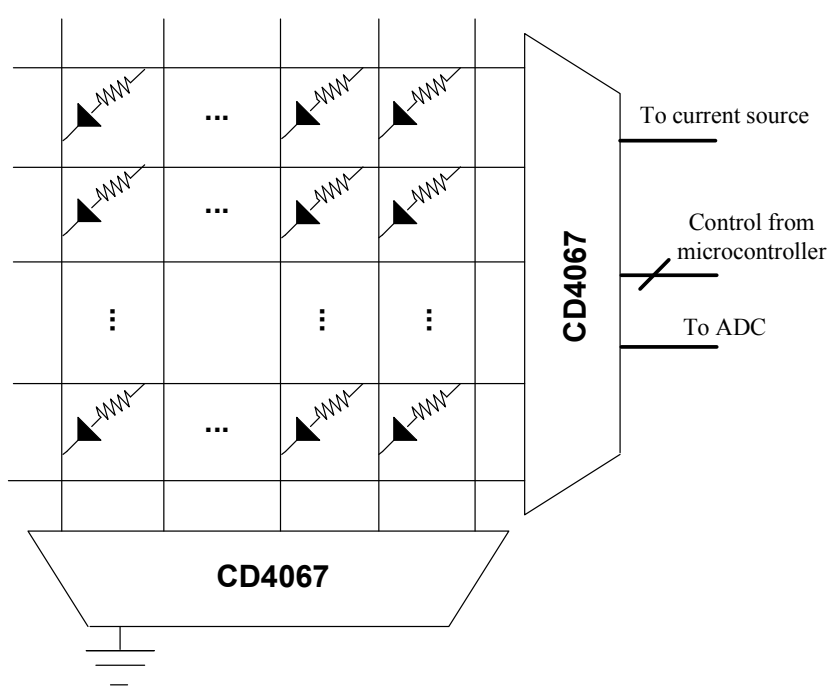

Figure 3: Schematic of resistive sensors array.

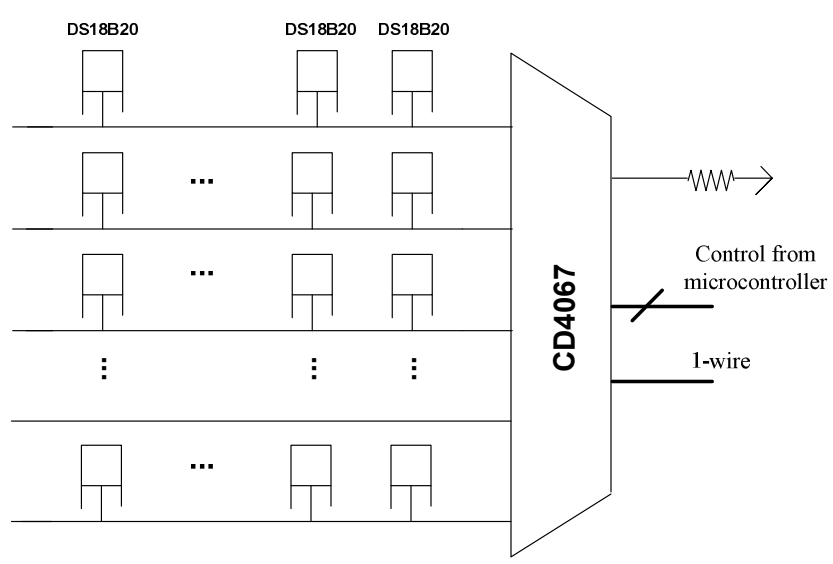

Figure 4: Schematic of temperature sensors arrangement.

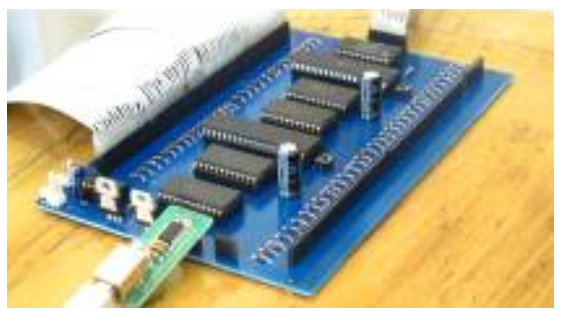

Figure 5: PCB used for the electronics.

\section{Software Design}

Digitized data of sensors is transmitted from the microcontrollers to a computer via two USB interfaces using FT232 chips. A GUI (Graphical User Interface) is developed in MATLAB to report pressure and temperature maps in real time, retrieve previous maps and risks and set alarms. Post processing of the obtained data is carried out in MATLAB.
Measured values of pressure and temperature of each sensor in the array are saved in matrices at each sampling interval. In the GUI, there is an option for the user to define two thresholds for pressure intensity and duration. An alarm can be created by the software if the pressure intensity of one sensor is larger than the adjusted threshold and the duration of that pressure is more than the time threshold. This event is considered as a risky situation. It is possible to observe the temperature map corresponding to the same risk situation to check whether there is any significant temperature change in areas experiencing excessive pressure. As mentioned earlier, temperature changes can be used for indicating pressure ulcer development.

The GUI is designed in a way that we can see the last risks and their occurrence times. This would also provide useful information regarding patient's postural history.

\section{EXPRIMENTAL RESULTS}

A sample pressure map of a person sitting on the setup is shown in Fig. 6. The GUI stores the pressure in units of $\mathrm{mmHg}$ for each sensor and MATLAB post processing is used to generate this pressure map. The temperature map of the same situation, after post processing, is given in Fig. 7.

As an experiment, a volunteer sat on the seat for 10 minutes. Pressure and temperature matrices were stored in MATLAB during this period. Time dependant behavior of specified pressure sensors is presented in Fig. 8 as pressure $[\mathrm{mmHg}]$ vs. time [s] graphs. We obtained pressure-time graphs corresponding to the locations in which temperature sensors are placed using interpolation. These graphs include the volunteer sitting and getting up instant as well.

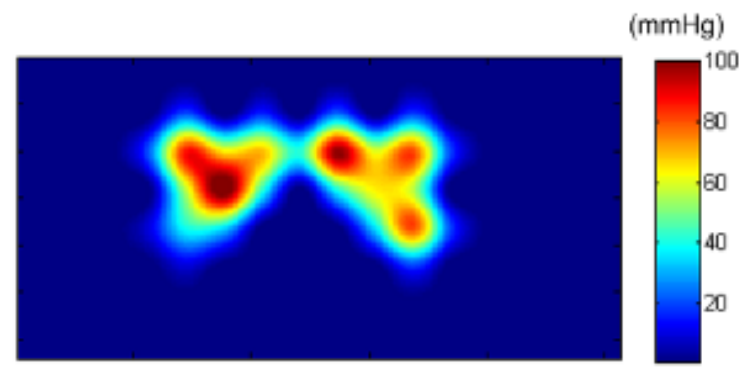

Figure 6: Pressure map of the sited person after MATLAB post processing. $\left({ }^{\circ} \mathrm{C}\right)$

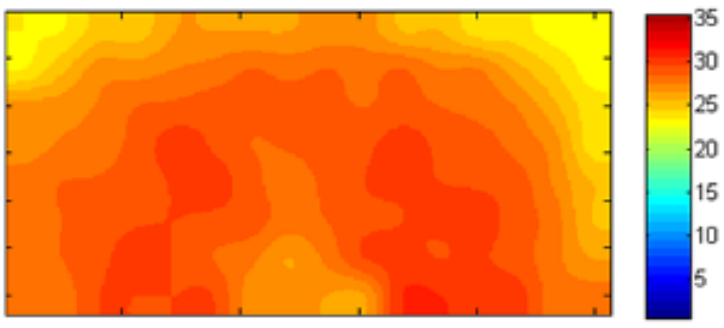

Figure 7: Temperature map of the sited person after MATLAB post processing. 

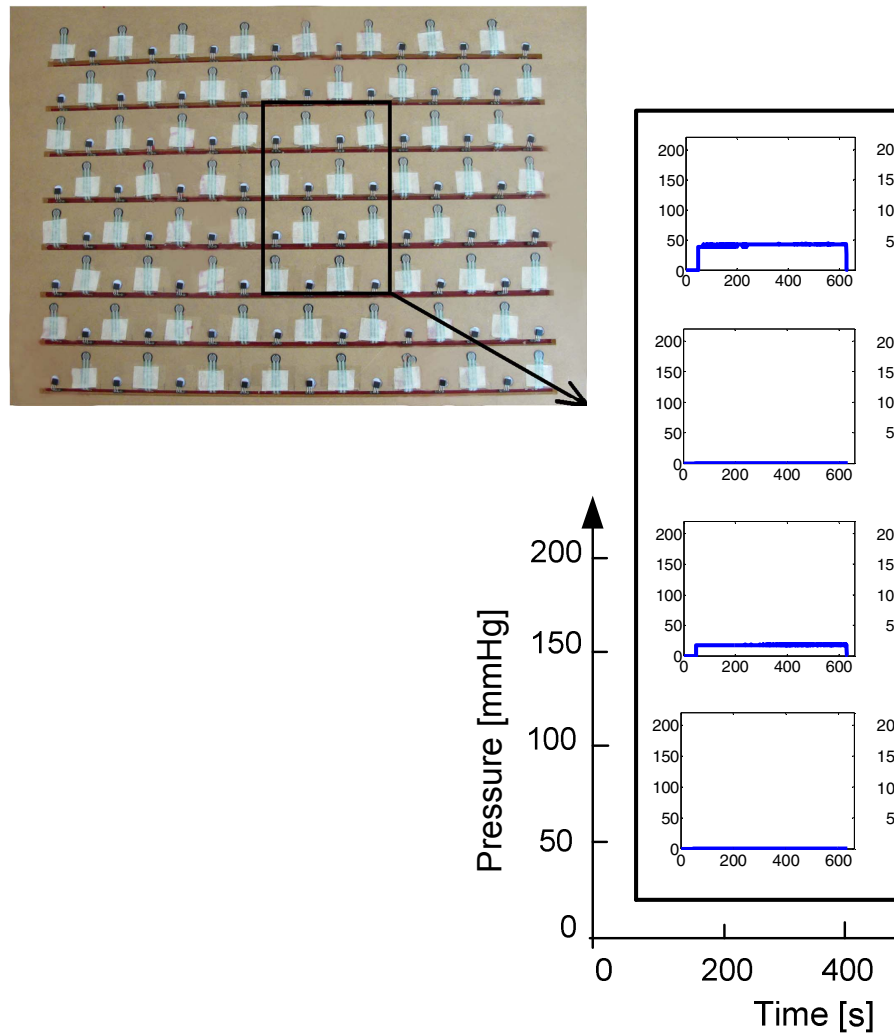

Time [s]
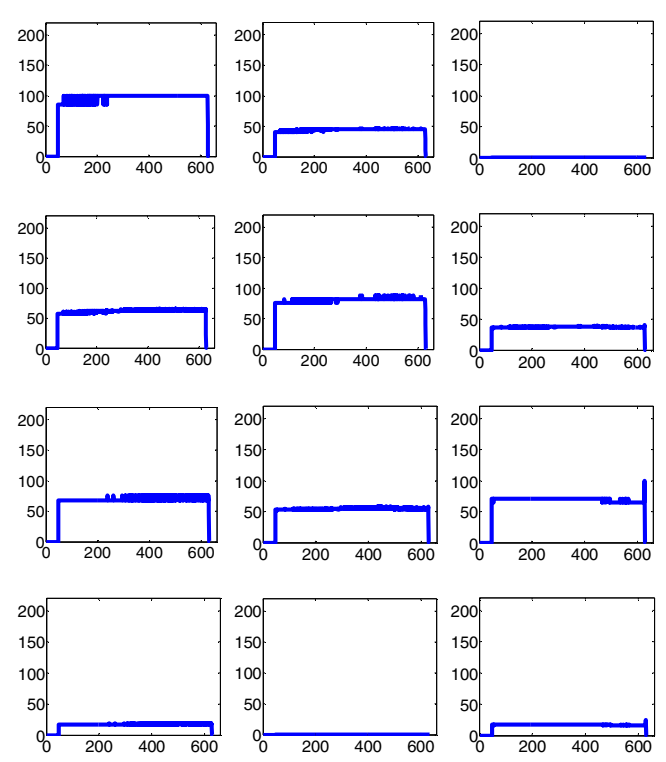

Figure 8: Pressure $[\mathrm{mmHg}]$ vs. time [s] characteristics of specified sensors during the test procedure. Characteristics corresponding to the locations, in which temperature sensors are placed, are obtained using interpolation.

Early abrupt pressure changes observed in Fig. 8 are due to preliminary repositioning of the subject. We calculated mean and standard deviation of measured pressures for each of these locations, excluding values related to the volunteer sitting and getting up. Results are shown in TABLE I. Small deviations in the recorded values during prolonged test sessions may be the results of subject's smooth movements on the setup.

Temperature vs. time characteristics of the specified temperature sensors are presented in Fig. 9. We obtained temperature-time graphs corresponding to the locations in which pressure sensors are placed using interpolation. A gradual rise in the recorded temperature values is observed at the contact locations.

\section{CONCLUSION}

A continuous-time pressure and temperature monitoring system has been presented. Due to its helpful information about patient's movement history, feasibility for simultaneous monitoring of pressure and temperature and alarming options, it is proposed that this system can be utilized for pressure ulcer prevention.

Further test protocols need to be designed and executed to investigate different characteristics of the system. Spatial resolution can be improved in future works by increasing the number of pressure sensors. Additional temperature sensors are not required, since temperature changes are gradual and therefore no significant change is expected in the measured values of adjacent sensors.

TABLE I: Mean and standard deviation of pressures measured for each location. Pressure values related to subject sitting and getting up is excluded.

\begin{tabular}{|l|c|c|}
\hline & Mean [mmHg] & Standard Deviation \\
\hline Row \#1,Col. \#1 & 42.3443 & 0.8866 \\
\hline Row \#1,Col. \#2 & 98.7963 & 3.3755 \\
\hline Row \#1,Col. \#3 & 45.1215 & 1.0344 \\
\hline Row \#1,Col. \#4 & 0 & 0 \\
\hline Row \#2,Col. \#1 & 0 & 0 \\
\hline Row \#2,Col. \#2 & 62.7561 & 1.6617 \\
\hline Row \#2,Col. \#3 & 81.6663 & 2.0484 \\
\hline Row \#2,Col. \#4 & 37.6311 & 0.7204 \\
\hline Row \#3,Col. \#1 & 17.6463 & 1.0552 \\
\hline Row \#3,Col. \#2 & 70.5500 & 4.2207 \\
\hline Row \#3,Col. \#3 & 55.2656 & 1.1388 \\
\hline Row \#3,Col. \#4 & 68.8344 & 2.5505 \\
\hline Row \#4,Col. \#1 & 0 & 0 \\
\hline Row \#4,Col. \#2 & 17.6463 & 1.0552 \\
\hline Row \#4,Col. \#3 & 0 & 0 \\
\hline Row \#4,Col. \#4 & 17.2174 & 0.6376 \\
\hline
\end{tabular}



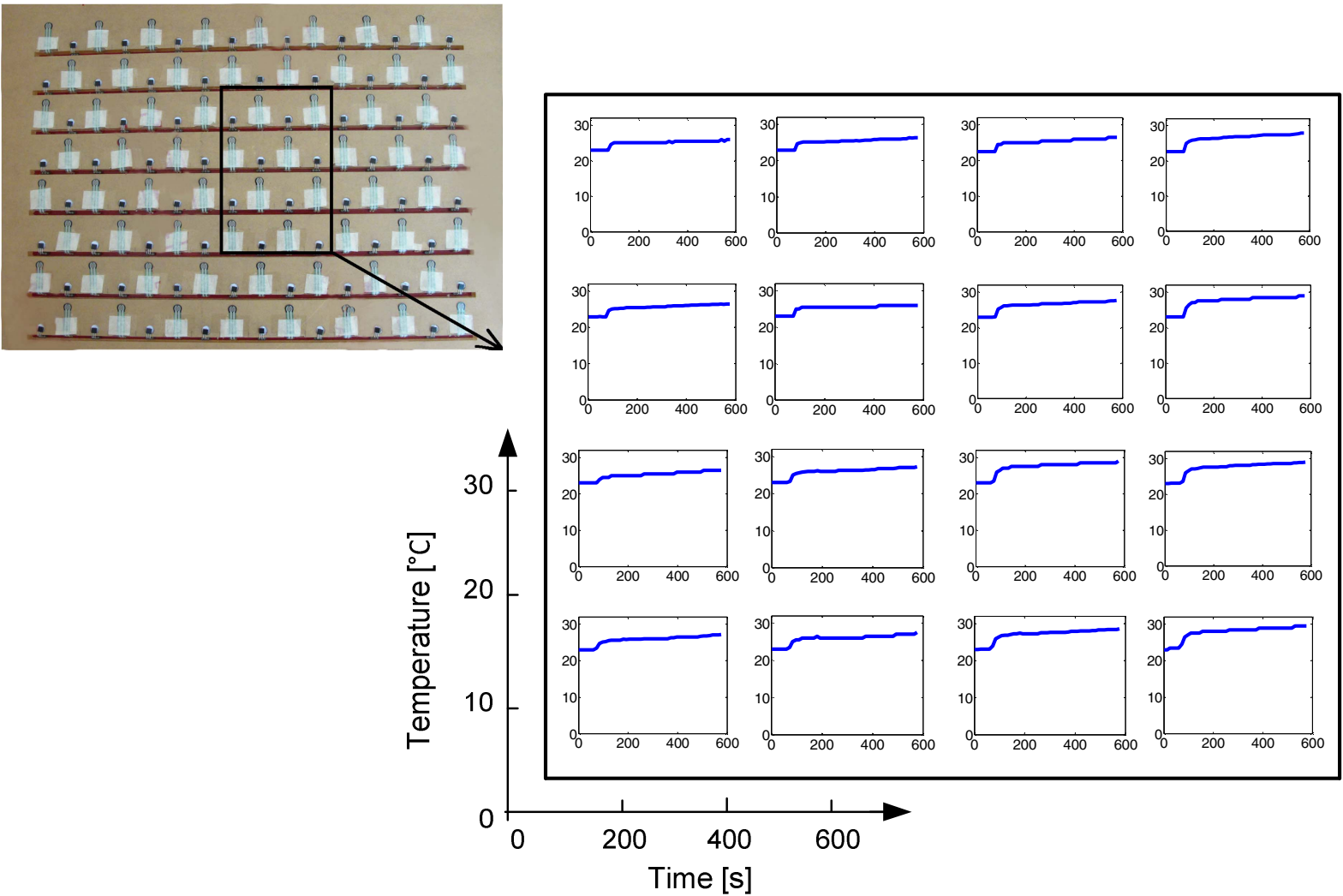

Figure 9: Temperature $\left[{ }^{\circ} \mathrm{C}\right]$ vs. time [s] characteristics of specified sensors during test period. Characteristics corresponding to the locations, in which pressure sensors are placed, are obtained using interpolation.

\section{ACKNOWLEDGMENT}

The authors would like to thank A. Sharif and S. Rahmanpour for their technical assistance.

\section{REFERENCES}

[1] M. Reddy, S.S. Gill and P.A. Rochon, "Preventing pressure ulcers: a systematic review," Journal of American Medical Association, vol. 296, pp. 974-984, Aug. 2006.

[2] J. Elliott. (2009 Mar.). How one nurse helped stop killers bedsores. News article, BBC News. [Online]. Available: http://news.bbc.co.uk/2/hi/health/7952519.stm

[3] J.B. Reswick and J.E. Roger, "Experience at Rancho Los Amigos hospital with devices and techniques to prevent pressure sores," in Bedsore Biomechanics, Baltimore: University Park Press, 1976, pp. 301-310.

[4] I. Swain, "The measurement of interface pressure," in Pressure ulcer research: Current and future perspectives, D. Bader, C. Bouten, D. Colin C.Oomens, eds. Berlin: Springer-Verlog, 2005, p.52.

[5] European Pressure Ulcer Advisory Panel (EPUAP) and National Pressure Ulcer Advisory Panel (NPUAP) (2009). Pressure Ulcer Prevention, Quick Reference Guide. [Online]. Available: http://www.npuap.org

[6] K. Sakai, H. Sanada, N. Matsui, G, Nakagami, J. Sugama, C. Komiyama and N. Yahagi, "Continuous monitoring of interface pressure distribution in intensive care patients for pressure ulcer prevention," Journal of Advanced Nursing, vol. 65, pp. 809-817, Apr.2009.

[7] R. Meffre, C. Gehin and A. Dittmar, "MAPI: Active interface pressure sensor integrated into a seat," in Proceeding of the $29^{\text {th }}$ annual international conference of the IEEE EMBS, pp. 1358-1361, 2007.
[8] M. Yip, D. Da He, E. winokur, A. G. Balderrama, R. Sheridan and H. $\mathrm{Ma}$, "A flexible pressure monitoring system for pressure ulcer prevention," in $31^{\text {st }}$ annual international conference of the IEEE EMBS, pp. 1212-1215, 2009.

[9] Y. Yang, J. Wang, Z. Gao and Y. Zhou, "Design and preliminary evaluation of an air-alternating wheelchair seating system for pressure ulcer prevention," in international conference on bioinformatics and biomedical technology, pp. 239-243, 2010.

[10] D.B. Drennan and D.W. Southard, "System and method of reducing risk and/or severity of pressure ulcers," U.S. Patent US2010/0268122 A1, Oct. 21, 2010.

[11] S. Sprigle, M. Linden, D. McKenna, K. Davis and B. Riordan, "Clinical skin temperature measurement to predict incipient pressure ulcers," Advanced in Skin and Wound Cares, vol. 14, No. 3, pp. 133-137, May/Jun 2001 